\title{
Die Gründe der «Kostenexplosion» im Gesundheitswesen
}

\author{
Seit zehn Jahren sammelt der Autor Listen aus Periodika, Büchern, Referaten und \\ im Internet mit Gründen für die «Kostenexplosion» im Schweizer Gesundheitswesen. \\ Am häufigsten genannt werden sieben Gründe. Eine erhebliche Rolle dürften aber \\ auch Gründe spielen, die übehaupt nicht erwähnt werden.
}

«Die Kostenexplosion bei Spitälern und Krankenkassen ist seit längerem ein vieldiskutiertes Thema ... Das Gesundheitswesen scheint ein Fass ohne Boden zu sein.» Dies schrieb ich 1975 in einem Artikel. In den 36 Jahren seither nahmen die Klagen über die Kostenentwicklung wellenförmig, aber ununterbrochen zu. Ein Übel sollte man bekanntlich an den Wurzeln bekämpfen. Die Kosten eines Gesundheitswesens sind aber multifaktoriell bedingt: Für die «Kostenexplosion» können unzählige Gründe (und Schuldige) aufgezählt werden. Einzelne Sparmassnahmen bringen wenig. Würde man die «wahren» Gründe und Ursachen kennen und dort ansetzen, wäre der Kostendämpfungseffekt grösser. In Politik und Medien werden laufend irgendwelche Kostentreiber und Schuldige an den Pranger gestellt. Wo überhaupt eine Begründung mitgeliefert wird, steht sie praktisch immer auf schwachen Füssen. Dies ist auch nicht verwunderlich, da ohne weiteres 200 und mehr Gründe für die «Kostenexplosion» aufgezählt werden können.

In dieser Situation ist es interessant, die herrschende Meinung der echten und auch der selbsternannten Fachleute über die Gründe der ständigen Kostenzunahmen zu kennen.

\section{Vorgehen}

Seit zehn Jahren sammle ich alle Listen mit Gründen resp. Ursachen der «Kostenexplosion», die ich in Periodika, Büchern, Referaten und im Internet fand. Nicht erfasst sind Listen, die nur die Krankenversicherung betreffen, da diese nur 35\% der nationalen Gesamtausgaben trägt, und weil die Kostenursachen dort teilweise anders sind. Die Listen stammen von Wissenschaftlern, Gesundheitspolitikern der Exekutive und Legislative, Interessenverbänden, Leistungsanbietern, Medienvertretern und anderen Personen.

\section{Ergebnisübersicht}

Die Sammlung ergab 65 Listen mit je 2 bis 23 Ursachen. 47 Listen stammen aus der Schweiz, 18 aus anderen Ländern. Im Durchschnitt wurden pro Liste 4,4 Gründe aufgeführt. Insgesamt nannten die Autorinnen und Autoren 292 Gründe/Ursachen für die Zunahme der Gesundheitsausgaben («Kostenexplo-

\section{Les motifs de I'«explosion des coûts» dans le domaine de la santé}

L'article se penche sur 65 travaux consacrés à l'évolution des coûts au cours de ces dernières années et nous indique que les causes les plus fréquemment évoquées sont les «progrès/innovations médicotechniques» ainsi que le «vieillissement, allongement de l'espérance de vie, évolution/changement démographique». Ces deux raisons sont mentionnées dans plus de $83 \%$ des cas. Au $3^{\text {e }}$ rang, on trouve les «exigences, le comportement et les attentes de la population/des patients» (37\%). Au $4^{\text {e }}$ rang figure la «densité accrue des médecins et des hôpitaux, offre élevée et en hausse» (23\%), suivie d'une augmentation de la prospérité (22\%) et d'«incitatifs manquants ou mauvais pour économiser, incitatifs erronés» (19\%).

Parmi les sept motifs les plus fréquemment évoqués, 4 sont influençables. Alors que dans les 3 autres cas, une intervention n'est pratiquement pas possible ou simplement pas souhaitée.

sion»). Da viele davon unterschiedlich formuliert das Gleiche meinen, konnten sie zu 75 Gründen aggregiert werden.

Die Tabelle zeigt die 22 am häufigsten genannten Ursachen. Zwei Gründe wurden überraschend deutlich als Hauptkostentreiber genannt. Diese eindeutigen Spitzenreiter wurden beide auf über $80 \%$ aller Listen aufgeführt: der «medizinisch-technische Fortschritt, Innovationen» und «Alterung, steigende Lebenserwartung, demografische Entwicklung/Wandel». Im dritten Rang wurden die Ansprüche der Bevölkerung auf 39\% der Listen genannt. Nur sieben Gründe (s. Abb. 1) sind auf mehr als 10\% der Listen 
Tabelle 1

Umfrage über die Gründe/Ursachen der «Kostenexplosion» im Gesundheitswesen.

\begin{tabular}{|c|c|c|c|c|}
\hline \multirow[t]{2}{*}{ Nr. } & \multirow[t]{2}{*}{ Grund/Ursache } & \multicolumn{2}{|c|}{ genannt auf } & \multirow{2}{*}{$\begin{array}{l}\text { Angebot }(A) \text { oder } \\
\text { Nachfrage }(\mathrm{N}) \text { ? }\end{array}$} \\
\hline & & Anzahl der Listen & Prozent der Listen & \\
\hline 1 & Medizinisch-technischer Fortschritt, Innovationen & 56 & 86,2 & A \\
\hline 2 & $\begin{array}{l}\text { Alterung, steigende Lebenserwartung, demografische(r) Entwicklung/ } \\
\text { Wandel }\end{array}$ & 54 & 83,1 & $\mathrm{~N}$ \\
\hline 3 & Ansprüche, Anspruchshaltung, Erwartungen der Bevölkerung/Patienten & 24 & 36,9 & $\mathrm{~N}$ \\
\hline 4 & Höhere Ärzte- und Spitaldichte, hohes und wachsendes Angebot & 15 & 23,1 & A \\
\hline 5 & Wachsender Wohlstand & 14 & 21,5 & $\mathrm{~A}+\mathrm{N}$ \\
\hline 6 & Fehlende oder falsche Sparanreize, Fehlanreize & 12 & 18,5 & A \\
\hline \multirow[t]{2}{*}{7} & $\begin{array}{l}\text { Medizinischer Überkonsum, Mengenausweitung durch Leistungs- } \\
\text { erbringer, unnötige Leistungen }\end{array}$ & 10 & 15,4 & $\mathrm{~A}+\mathrm{N}$ \\
\hline & 7 häufigste Gründe & 185 & & \\
\hline 8 & $\begin{array}{l}\text { Zu grosser Leistungskatalog (Grundversicherung), Ausdehnung } \\
\text { der Versicherungsdeckung }\end{array}$ & 7 & 10,8 & A \\
\hline 9 & Wachsende Spezialisierung und Technisierung, Apparatemedizin & 6 & 9,2 & A \\
\hline 10 & Verbesserter Zugang zu qualitativ hochstehender Behandlung & 6 & 9,2 & A \\
\hline 11 & Zunahme chronischer Krankheiten & 5 & 7,7 & $\mathrm{~N}$ \\
\hline 12 & Föderalismus, «Kantönligeist» & 4 & 6,2 & A \\
\hline 13 & Medikalisierung gesellschaftlicher Probleme & 4 & 6,2 & A \\
\hline 14 & Immer teurere Medikamente & 4 & 6,2 & A \\
\hline \multirow[t]{2}{*}{15} & Bevölkerungszunahme & 4 & 6,2 & $\mathrm{~N}$ \\
\hline & 15 häufigste Gründe & 225 & & \\
\hline 16 & Immer teurere Forschung und Spitzenmedizin & 2 & 3,1 & A \\
\hline 17 & Kurative statt präventive Medizin, Prävention unterentwickelt & 2 & 3,1 & A \\
\hline 18 & Kommerzialisierung & 2 & 3,1 & A \\
\hline 19 & Verwaltungsaufwand & 2 & 3,1 & A \\
\hline 20 & $\begin{array}{l}\text { Produktivitätssteigerung bei Dienstleistungen nur beschränkt möglich } \\
\text { (Baumol) }\end{array}$ & 2 & 3,1 & A \\
\hline 21 & Allgemeine Teuerung & 2 & 3,1 & A \\
\hline \multirow[t]{2}{*}{22} & Nachfragesteuerung durch die Angebotsseite & 2 & 3,1 & A \\
\hline & 22 häufigste Gründe & 239 & & \\
\hline 53 & Restliche 53 Gründe, die je nur einmal genannt wurden & 53 & 1,5 & \\
\hline 75 & Total genannte Gründe/Ursachen (aggregiert) & 292 & & \\
\hline
\end{tabular}

aufgeführt. 53 der 75 Gründe wurden nur einmal genannt (Liste beim Autor erhältlich).

\section{Das Angebot, nicht die Nachfrage ist entscheidend}

Von den 15 meistgenannten Gründen liegen 60\% (9 Gründe) auf der Angebotsseite, 27\% (4) bei der Nachfrage, und 13\% (2) können beiden Seiten zugerechnet werden. Die 22 meistgenannten Gründe kamen alle auf zwei oder mehr Nennungen. Von diesen Gründen betrafen sogar 73\% die Angebotsseite. Auf die Nachfrageseite entfielen hier 18\%, auf beide Seiten 9\%.

Dies entspricht der vorherrschenden Erkenntnis in der gesundheitsökonomischen Fachliteratur: Im Gesundheitsmarkt dominiert das Angebot und nicht die Nachfrage, die stark angebotsinduziert ist. Oft genannt, aber ohne zuverlässige Quellenangabe ist die Aussage, dass die Gesundheitsausgaben zu rund 70 Prozent von Ärzten und anderen Leistungsanbietern «verursacht» oder zumindest bestimmt werden. Stichwörter dazu sind: «Therapiefreiheit», Ärztemonopol für zahlreiche Therapien, Behandlungen durch den Arzt selbst, Verschreibung und Selbstdispensation von Medikamenten, Anordnung weiterer diagnostischer und therapeutischer Abklärungen und Behandlungen, Spitaleinweisungen, Krankschreibung usw. Sparmassnahmen müssen deshalb vor allem auf die Struktur des Angebots und die Beeinflussung der Leistungserbringer zielen und nur sekundär auf das Patientenverhalten im Krankheitsfall. 


\section{Abbildung 1}

Die sieben Hauptgründe der «Kostenexplosion»: Zahl der Nennungen in Prozent aller Listen.

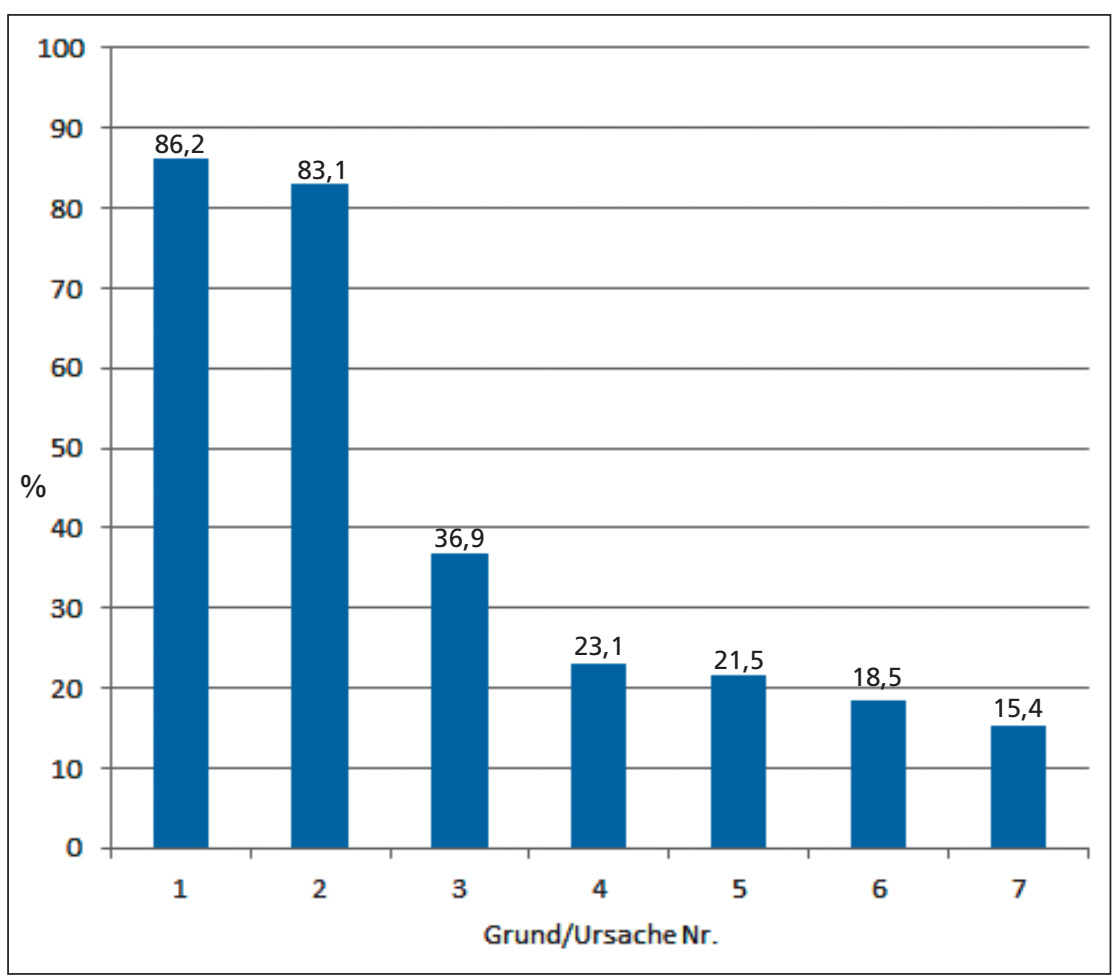

\section{Kurzkommentare zu den sieben meist- genannten Gründen}

1. Rang, aufgeführt in $86 \%$ der Listen: medizinisch-technischer Fortschritt, Innovationen Dies ist tatsächlich ein Hauptgrund. Wenn die moderne Medizin weiterhin so grossartige Fortschritte macht, werden wir bald alle alt, krank und pleite sein. Den Stand der medizinischen Versorgung von 1980 oder 1990 könnten wir uns locker leisten. Dass Fortschritte die Produkte verteuern, ist aber keineswegs selbstverständlich: Paradebeispiele sind Computer und Autos, bei denen eklatante Qualitätsfortschritte mit erheblich tieferen Preisen verbunden sind. Die Mehrausgaben für das Gesundheitssystem dagegen unterliegen dem «Gesetz des abnehmenden Grenznutzens».

Im Gesundheitswesen gibt es eine grosse Diskrepanz: Behandlungsfortschritte und Produktinnovationen sind häufig und breiten sich rasch aus. Die Gesundheitsversorgung dagegen klebt an alten Zöpfen wie 26 kantonalen Gesundheitspolitiken im Zwergstaat Schweiz, überflüssigen Krankenhausbetten, Einzelpraxen, Selbstdispensation und überholten Medikamenten.

2. Rang, aufgeführt in $\mathbf{8 3} \%$ der Listen: Alterung, steigende Lebenserwartung, demografische $(r)$ Entwicklung/Wandel

Hier ist ein Doppeleffekt festzustellen: Altersstruktur der Bevölkerung (mehr Alte) und höhere Kosten pro alter Mensch. Allerdings wissen alle Fachleute seit über 20 Jahren, dass der Einfluss dieser Faktoren auf die Gesundheitskosten weit überschätzt wird. Es gibt dazu Dutzende von seriösen Studien, aber wer liest sie schon - das Argument Alterung ist zu einleuchtend. Vorgebracht wird es nur allzu gerne von den Berufen und Institutionen, die als Kostentreiber und häufig als Abzocker angegriffen werden. Ein Grund für die Überschätzung der von den Alten verursachten Kosten liegt darin, dass zu oft nur die Ausgaben der Krankenversicherung beachtet werden und nicht auch die doppelt so hohen Ausgaben, welche anders finanziert werden. Bei diesen sind die altersbedingten Kosten erheblich tiefer.

\section{Rang, aufgeführt in $\mathbf{3 7} \%$ der Listen: Ansprü- che, Anspruchshaltung, Erwartungen der Bevölkerung/Patienten}

Wenn die Schuld den «begehrlichen» Patienten zugeschoben werden soll, ist zu fragen: Wer steigert die Erwartungen der Bevölkerung? Doch in erster Linie die Medizin, die Mediziner, die Pharmaindustrie, mit tatkräftiger Unterstützung vieler Medien. Das Konsumdenken wird heute überall geschürt, auch in der Medizin, und Anspruchshaltung ist auch Anbietern nicht fremd ... Warum sollte der Mensch, der im ganzen Leben systematisch auf Konsummaximierung getrimmt wird, ausgerechnet dann anspruchslos sein, wenn er krank ist? Die bestmögliche Behandlung zu wollen, gehört zum Genesungswillen und darf kein Grund sein, den Kranken zu verurteilen (victim blaming). Was aber gemacht werden kann: übertriebene Erwartungen und Illusionen über die moderne Medizin dämpfen. Übrigens auch im wohlverstandenen Interesse der Behandler selbst.

\section{Rang, aufgeführt in $23 \%$ der Listen: höhere Ärzte- und Spitaldichte, hohes und wachsendes Angebot}

Das ist zweifellos ein wichtiger Grund der Kostenentwicklung, wie bereits aus den Ausführungen zur angebotsinduzierten Nachfrage hervorgeht. Ein alter Gesundheitsökonomenspruch lautet: "A built bed is a filled bed and a filled bed is a billed bed.» Bei den überflüssigen Spitälern, Betten und Aufenthaltstagen werden aber die Fallpauschalen (DRG) Remedur schaffen. Die Spitäler und Heime sind mit 52\% der nationalen Gesundheitsausgaben der weitaus grösste Kostenblock im Gesundheitssystem. Daher wird dank Fallpauschalen der Überfluss an stationärer Behandlung als ein Haupt-Kostentreiber an Gewicht verlieren.

\section{Rang, aufgeführt in $22 \%$ der Listen: wachsender Wohlstand}

Dies ist ein weniger offensichtlicher, aber zentraler Grund (für manche sogar der Hauptgrund für die Zunahme der Gesundheitsausgaben). Die reichen Länder geben pro Einwohner nicht nur frankenmässig, sondern auch im Verhältnis zum Pro-Kopf-Bruttosozialprodukt mehr für die Gesundheitsversorgung aus als die weniger reichen Länder. In der Ökonomie wird Gesundheit als ein superiores Gut bezeichnet, das heisst als ein Gut, das mit zunehmendem Ein- 
kommen überproportional stark nachgefragt wird (Einkommenselastizität der Nachfrage grösser als 1). In Grafiken mit dem Ländervergleich der Pro-KopfGesundheitsausgaben und des BSP pro Kopf zeigt sich diese Korrelation deutlich.

\section{Rang, aufgeführt in $19 \%$ der Listen: fehlende} oder falsche Sparanreize, Fehlanreize

Dass dieser Grund nur auf 12\% der Listen genannt wird, ist wenig im Verhältnis zur Bedeutung dieser Ursache. Gesundheitsökonomen schätzen diesen Kostentreiber als viel verheerender ein. In ihrer Rangliste wäre er nicht nur im sechsten Rang (mit 12 von 66 Stimmen). Wie man die Körner streut, so wandern die Hühner ... Gesundheitsökonomen haben zwar oft die Berufskrankheit, den Menschen nur als homo oeconomicus zu sehen, der kein anderes Ziel als die Maximierung des Einkommens und der Gewinne kennt. Dieses primitive Menschenbild ist längst als falsch entlarvt worden.

Unbestreitbar ist aber, dass es auch im Gesundheitswesen Behandler und Organisationen gibt, für die der finanzielle Aspekt im Vordergrund steht (Tendenz steigend). Die Medizin ist natürlich nicht ein Bereich, der nur Menschenliebe und Idealismus kennt. There is no business like health business! Zahllose Finanzanreize im Gesundheitswesen fördern diese Fehlentwicklung Richtung Umsatzbolzerei und Gewinnmaximierung und führen Betreiber von Gesundheitseinrichtungen und Behandler täglich in Versuchung. Eine schweizerische Spezialität dafür ist der Medikamentenhandel in der Arztpraxis, auch Selbstdispensation genannt.

Zentrales Instrument für richtige Finanzanreize sind die Tarife, die der Schweizer «Tarif-Bapst» kürzlich in zwei monumentalen Bänden im Detail analysiert hat [1]. Was im Tarifsektor passiert oder nicht passiert, ist für die Behandlungs- und Kostensteuerung mindestens so wichtig wie das endlose Herumschrauben am KVG. Die Erkenntnis wächst, dass das Gesundheitssystem über Tarife präziser gesteuert werden kann, und erst noch mit weniger Politisiererei.

\section{Rang, aufgeführt in $15 \%$ der Listen: medizini- scher Überkonsum, Mengenausweitung durch Leistungserbringer, unnötige Leistungen}

Trotz der Nennungen auf nur 15\% der Listen ist dieser Kostenfaktor absolut zentral. Das Gesundheitswesen ist immer noch - trotz aller Sparmassnahmen ein Reich der Verschwendung. Praktisch jede Diagnostik und Therapie kann ohne Notwendigkeit ausgeweitet, verlängert und verteuert werden, nicht zuletzt dank der «Therapiefreiheit» und fehlender Kontrollen. Überversorgung ist als Problem mindestens so wichtig wie Unter- oder Fehlversorgung. Untersuchungen von regionalen Häufigkeitsunterschieden aller möglicher Behandlungen und diagnostischer Massnahmen füllen ganze Bände (s. www. dartmouthatlas.org). Oft ist eine Behandlung in einer
Region drei- oder auch zehnfach häufiger als in einer völlig vergleichbaren anderen Region. Solche Unterschiede sind medizinisch nicht zu erklären. Sie zeigen ein grosses Sparpotential auf.

\section{Welche Gründe wurden nicht genannt?}

Die Listen enthalten insgesamt 292 Gründe und Ursachen, die hier zu 75 Clusters aggregiert wurden. Man könnte annehmen, dass damit praktisch alle möglichen Gründe aufgeführt sind. Dies ist aber nicht der Fall. Nie genannt sind beispielsweise schlechtes Management in Exekutiven und Legislativen und im Gesundheitssystem (Einrichtungen, Ämter, Unternehmen, Verbände); ungenügende wissenschaftliche Analysen des Gesundheitswesens, ungenügende Politikberatung, keine Ziel- und Strategiediskussion, Lobbying. Das gleiche gilt für die Aus-, Weiter- und Fortbildung - das Wort Bildung wird in den 292 aufgeführten Gründen kein einziges Mal auch nur erwähnt.

Sehr wenige Nennungen entfallen auf andere wichtige Kostenursachen: etwa unseren Extremföderalismus mit zahlreichen Parallelstrukturen, überforderten Kleinorganismen und ungenügender Koordination. Dazu kommt die Kommerzialisierung mit Kostentreibern wie überflüssigen Leistungen, teuren Medikamenten und einer wahren Marketinglawine Richtung Maximalmedizin. Wenig genannt werden auch die starke Medikalisierung aller möglicher Probleme (La santé n'existe pas!) und nur schwach entwickelte Bereiche wie die Prävention und die Qualitätsförderung.

\section{Fazit}

Es gibt keine allgemein anerkannte Rangliste der Kostengründe und -ursachen. Eine solche Rangierung mit wissenschaftlichen Methoden wäre auch nicht möglich. Die vorliegende Zusammenstellung gibt aber nützliche Hinweise für Sparmassnahmen. Von den sieben meistgenannten Gründen sind vier gut beeinflussbar (Gründe 3, 4, 6 und 7). Bei den anderen drei Gründen (1,2 und 5) ist Einflussnahme kaum möglich oder nicht wünschbar.

Dass die Schweiz trotz aller Sparrhetorik Jahr für Jahr über 2 Milliarden Franken mehr ausgibt als noch im Vorjahr, ist ein Skandal. Über die Ausgabenentwicklung im Gesundheitssystem wird seit mehr als 36 Jahren ununterbrochen geklagt. Gleichwohl sind Sparerfolge selten, und dieses Jahr geben wir fast 65 Milliarden Franken für das Gesundheitswesen aus. Resignation, Defaitismus und Zynismus herrschen weitherum vor. Ziel des Gesundheitswesens ist nicht das Sparen - das Verschwenden aber auch nicht. Der ökonomische und politische Druck nehmen zu, die Hauptursachen der Dauerexplosion sind bekannt, die Hauptschuldigen auch. Ein vorsichtiger Optimismus, dass die 2 Milliarden Franken Zunahme pro Jahr reduzierbar sind, ist keine Illusion mehr. Sollte man

meinen.
Macht. 2 Bde.

Bern: Verlag SGGP; 2010 